\title{
CALIDAD DE VIDA RELACIONADA CON SALUD. INSTRUMENTOS DE MEDICIÓN PARA VALORACIÓN DE RESULTADOS EN CIRUGÍA DIGESTIVA ALTA*
}

\author{
Drs. Carlos Manterola D. ${ }^{1,2}$, Sebastián Urrutia V. ${ }^{1}$, Tamara Otzen H. ${ }^{3,4}$ \\ 1 Departamento de Cirugía, Universidad de La Frontera. \\ 2 Centro de Investigación en Ciencias Biomédicas, Universidad Autónoma de Chile. \\ 3 Programa de Doctorado en Ciencias Médicas, Universidad de La Frontera, Chile. \\ 4 Escuela de Psicología, Universidad Autónoma de Chile. \\ Chile.
}

\begin{abstract}
Health-related quality of life. Measurement tools to assessing upper gastrointestinal surgery outcomes
\end{abstract}

Background: Since a while, clinicians and surgeons recently have recognized the desirability of Health-related quality of life (HRQOL) measure to inform their patients, even health authorities in respect of decision making in health and as a variable to be considered in clinical research. For this, there are multiple questionnaires (including self-administered) that are available for measuring this construct. In the field of upper gastrointestinal surgery, there are tools that have been generated and validated for this purpose (some of them generals and other specifics). The aim of this article is to describe specific measurement instruments to assess HRQOL in upper gastrointestinal surgery. Material and Methods: A search on engines Altavista, Google and Yahoo, in Ixquick and Copérnico metasearch and in the database "Documents in Information Science" (DoIS) was performed. The search strategy used the terms "Quality of Life", "Health-related quality of life", "HRQOL", "Outcome Assessment (Health Care)", "upper gastrointestinal surgery" and "digestive surgery". After locating articles, these were evaluated and a summary document of collected information was developed. Results: The search performed gave 23 instruments: related with upper gastrointestinal benign disease (10), with obesity and bariatric surgery (4), and related with digestive neoplasms (9). Conclusion: There is an interesting variety of instruments to measure HRQOL in upper gastrointestinal surgery. Knowing is a user support to both the development of everyday surgical practice, as for decision-making in health and as measurement instruments in clinical research.

Key words: "Quality of Life"[Mesh], "Outcome Assessment (Health Care)", "Digestive System Surgical Procedures"[Mesh], Evidence-Based Medicine”[Mesh].

*Recibido el 4 de noviembre de 2013 y aceptado para publicación el 25 de noviembre de 2013.

Los autores no refieren conflictos de interés.

Correspondencia: Dr. Carlos Manterola D. carlos.manterola@ufrontera.cl 


\section{Resumen}

Antecedentes: Desde hace un tiempo a esta parte, los clínicos y últimamente los cirujanos han reconocido la conveniencia de medir la calidad de vida relacionada con salud (CVRS) para informar a sus pacientes y a las autoridades sanitarias respecto de la toma de decisiones en salud y como variable a considerar en investigación clínica. Para ello, existen múltiples cuestionarios (incluso auto-administrados) que se encuentran disponibles para medir este constructo. En el ámbito de la cirugía digestiva alta, existen instrumentos que se han generado y validado con este propósito (algunos generales y otros de carácter específico). El objetivo de este artículo es describir instrumentos de medición específicos para valorar CVRS en cirugía digestiva alta. Material y Método: Se realizó una búsqueda en los motores Google y Yahoo; en los metabuscadores Ixquick y Copérnico; y en la base de datos "Documents in Information Science" (DoIS). En la estrategia de búsqueda, se utilizaron los términos "calidad de vida", "calidad de vida relacionada con salud", "CVRS", "cirugía digestiva alta" y "cirugía gastrointestinal". Una vez localizados los resúmenes de los artículos localizados, se evaluaron los documentos en extenso y se desarrolló un documento resumen de la información recolectada. Resultados: La búsqueda realizada arrojó un total de 23 instrumentos; algunos relacionados con enfermedades benignas del tubo digestivo alto (10), otros relacionados con obesidad y cirugía bariátrica (4); y otros concernientes a cáncer del tubo digestivo alto (9). Conclusión: Existe una variedad interesante de instrumentos para medir CVRS en cirugía digestiva alta. Conocerlos constituye una ayuda al usuario tanto para el desarrollo de su práctica quirúrgica cotidiana, la toma de decisiones en salud y como instrumentos de medición en investigación clínica.

Palabras clave: Calidad de vida, calidad de vida relacionada con salud, cirugía digestiva alta, cirugía gastrointestinal, medicina basada en evidencias.

\section{Introducción}

El concepto de "calidad de vida" (CV) en el ámbito sanitario comenzó a utilizarse en 1947, con Karnofsky y Burchenal, quienes midieron este constructo en estudios de quimioterapia para el tratamiento de enfermedades neoplásicas ${ }^{1}$. Posteriormente, en 1952 fue definida por la OMS y comenzó a considerarse como variable a estudio por investigadores clínicos, por su relevancia como medida final de resultado de una intervención, al permitir la valoración del acto médico desde la visión del protagonista, el propio paciente ${ }^{2}$.

Nació luego, el concepto de "calidad de vida relacionada con la salud" (CVRS): "efecto funcional que una enfermedad o evento de interés y su tratamiento producen sobre un paciente o sujeto, tal como es percibido por éste". Los dominios o dimensiones que determinan y definen este concepto incluyen: Funcionalidad física y ocupacional; estado emocional; interacción social y percepción somática ${ }^{3}$.

Los estándares para medir y comparar efectividad en investigación en CVRS, a través de ensayos clínicos, fueron establecidos en 2012 por la Sociedad internacional de investigación en calidad de vida (ISOQOL); consenso en el que se hizo especial mención al concepto patient reported outcomes (PRO) o resultados reportados por el paciente ("cualquier reporte del estado de salud de un paciente que proviene directamente de él, sin la interpretación de un clínico o de terceros") $)^{4}$.
Sin embargo, en la medición de CVRS existen ciertos problemas metodológicos relacionados con la validez y sensibilidad de la medición y el diseño de los estudios; lo que determina la necesidad de observar con cautela la interpretación de los resultados. Es así como existen diferentes enfoques de medición de CVRS y por ende distintos tipos de instrumentos de medición; destacando los genéricos y los específicos, cada cual con sus ventajas y desventajas.

El objetivo de este artículo es describir instrumentos de medición específicos para valorar CVRS en cirugía digestiva alta.

\section{Material y Método}

Se realizó una búsqueda en los metabuscadores Ixquick y Copérnico, en los motores de búsqueda Google y Yahoo; y en la base de datos Documents in Information Science (DoIS) ${ }^{6}$; en el período marzo a septiembre de 2013.

Se utilizaron los términos "Quality of Life", "Health-related quality of life", "HRQOL", "Outcome Assessment (Health Care)", "upper gastrointestinal surgery" and "digestive surgery"; y los operadores booleanos AND y OR.

La lectura y extracción de datos de los diversos sitios encontrados fue realizada de forma independiente por los autores. Se generaron sesiones vía correo electrónico de los borradores con información recuperada, a partir de los cuales se desarrolló el documento final; el que fue discutido en sesiones presenciales. 
De forma arbitraria y sólo para los efectos de organización del material obtenido, este fue clasificado en instrumentos relacionados con enfermedades benignas del tubo digestivo alto, relacionados con obesidad y cirugía bariátrica, y concernientes a cáncer del tubo digestivo alto.

\section{Resultados}

La búsqueda realizada arrojó un total de 23 instrumentos y módulos adicionales. Diez relacionados con enfermedades benignas del tubo digestivo alto; 4 con obesidad y cirugía bariátrica; y 9 concernientes a cáncer del tubo digestivo alto.

Estos, se describen a continuación por orden alfabético en los subgrupos correspondientes.

\section{Relacionados con enfermedades benignas del tubo digestivo alto}

\section{GERD-HRQoL}

Diseñado por académicos del Departamento de Cirugía del Hospital Henry Ford, para medir CV en pacientes con enfermedad por reflujo gastroesofágico (ERGE). Está compuesto por 9 ítems (6 enfocados a pirosis, 2 a deglución y una al impacto de la terapia farmacológica diaria); y cada uno de ellos, tiene una escala entre 0 a 5 puntos, siendo 0 ausencia de síntomas, 1 síntomas presentes pero no molestos, 2 síntomas presentes y molestos pero no todos los días, 3 síntomas presentes y molestos todos los días, 4 síntomas que afectan las actividades diarias; y 5, síntomas incapacitantes que imposibilitan realizar las actividades diarias. El mejor puntaje total es 0 (asintomático) y el máximo puntaje posible es 45 (síntomas incapacitantes), significando un alto impacto en $\mathrm{CV}^{7}$. El cuestionario final incluye 5 preguntas adicionales; una concerniente al uso de medicamentos y las otras 4 enfocadas en los nuevos síntomas que pueden aparecer posteriores a la cirugía (disfagia, incapacidad para vomitar, eructar y sensación de acumulación de gas estomacal). Estas, fueron revisadas y aceptadas por gastroenterólogos y cirujanos con experiencia en funduplicatura laparoscópica. Cada respuesta se dispuso en escalas, con un puntaje que oscila entre 0 y 100 , donde un mayor puntaje representa mayor gravedad de síntomas y peor CV. Con esto se desarrollaron dos versiones del cuestionario; una de 45 preguntas para evaluación preoperatoria y otro de 50 preguntas para la aplicación postoperatoria, los cuales son idénticos a excepción de las 5 preguntas agregadas concernientes a las complicaciones y eficacia de la cirugía.

\section{GERD-QoL}

Diseñado y validado por académicos del Instituto de enfermedades digestivas de la Universidad de
Hong Kong, para medir CV en pacientes con ERGE. Se encuentra traducido al inglés. Compuesto por 16 ítems agrupados en 4 dominios (actividad diaria, efectos del tratamiento, dieta y bienestar sicológico $)^{8}$; que demostraron adecuada consistencia interna y confiabilidad.

\section{GERDyzer}

Diseñado por académicos del Departmento de Medicina de la Universidad de Adelaida, para medir $\mathrm{CV}$ en pacientes con ERGE; y validado a través de un estudio multicéntrico (Australia, Alemania y Sud África) en 20099. Está compuesto por 17 ítems que se distribuyen en 2 dominios (CV y nivel de satisfacción); cada una de las cuales se ilustra mediante dibujos que se evalúan mediante una escala visual análoga que va de 0 a 100; lo que permite una mejor comunicación con los pacientes. Se aplicó estudios de consistencia interna (alfa de Cronbach de 0,95), validez de constructo y confiabilidad test-retest (coeficiente de correlación intraclase de 0,91); en una población de pacientes con esofagitis. Es un instrumento autoadministrado.

\section{GIQLI (Gastrointestinal Quality of Life Index)}

Creado y validado por académicos del Departamento de Cirugía de la Universidad de Colonia, entre 1993 y 1995. Es un cuestionario bilingüe (alemán e inglés), y auto-administrado; que proporciona información sobre aspectos genéricos de CV y de síntomas digestivos del tracto superior e inferior, lo que permite que sea empleado en diferentes patologías y en la evaluación del funcionamiento de tratamientos médicos o procedimientos quirúrgicos. $\mathrm{Al}$ contar con áreas genéricas y específicas permite prescindir de un cuestionario genérico. Consta de 36 ítems que evalúan síntomas, disfunción emocional, física, social y respuesta a tratamiento médico. Las escalas de respuesta van de 0 a 4 (peor a mejor). La puntuación de cada área es la división del número de puntos de esa área por el número total de preguntas de la misma. La puntuación total se obtiene sumando las puntuaciones de cada pregunta ${ }^{10}$. Se encuentra traducido al español ${ }^{11}$.

\section{GSRS (Gastrointestinal Symptom Rating Scale)}

Diseñado por académicos del Departamento de Psiquiatría de la Universidad de Göteborg, en 1988 para evaluar la efectividad de diversos tratamientos para úlcera péptica y síndrome de intestino irritable. Surgió a partir de dos ensayos clínicos que comparaban el efecto de la psicoterapia individual y la terapia farmacológica para ambas patologías ${ }^{12,13}$. Fue desarrollada en analogía a la "Comprehensive Psychopathological Rating Scale" o escala de valoración integral psicopatológica ${ }^{14}$. Consta de 15 ítems 
(entre los que se encuentran dolor abdominal, sensación quemante, regurgitación ácida, distensión abdominal, náuseas y vómitos, sensación de evacuación incompleta, etc.). A todos los ítems se les asigna un puntaje basado en intensidad, frecuencia, duración e impacto en el diario vivir que este causa; que puede fluctuar entre 0 y 3 puntos, siendo 0 ausencia de síntomas y 3 sintomatología intensa. La confiabilidad interobservador es superior a 0,85 tanto para ítems como para los síndromes. Sin embargo, no cuenta con estudios de validación de contenido, criterio ni constructo; sólo existe evidencia de traducciones de la escala a otros idiomas.

\section{FDDQL (Functional Digestive Disorders Quality of life Questionnaire) ${ }^{15}$}

Diseñado por investigadores del Servicio de Medicina del Hospital Lariboisière, para evaluar respuesta al tratamiento en pacientes con dispepsia y síndrome del intestino irritable. $\mathrm{Su}$ proceso de validación se realizó de forma conjunta en Alemania, Francia y Reino Unido, por lo que se encuentra traducido a tres idiomas. Cuenta con determinación de consistencia interna (alfa de Cronbach de 0,94), validez discriminante y concurrente. Está compuesto por 43 ítems distribuidos en 8 dominios (actividades diarias, ansiedad, dieta, sueño, discomfort, capacidad para enfrentar la enfermedad, control de la enfermedad y estrés).

\section{QOLARS (Quality-of-Life Questionnaire for patients undergoing Antireflux surgery)}

Creado en la Semmelweiss University en 2005 con el propósito de medir la eficacia y éxito de la cirugía antirreflujo (desde el punto de vista de los pacientes y su percepción del impacto de esta en su CV $)^{16}$. Está compuesto por 50 ítems e incluye escalas validadas genéricas y específicas para $\mathrm{CV}$ en ERGE. Para la medición de CV se utiliza el cuestionario EORTC QLQ-C30 y como instrumentos específicos se utilizaron el cuestionario GERD-HRQoL ${ }^{7}$ modificado y la escala de Visick ${ }^{17}$.

\section{QOLRAD (Quality of life in reflux and dyspepsia)}

Diseñado en 1998 por investigadores del Astra Hässle AB, Mölndal, para la evaluación de CV en pacientes con ERGE y dispepsia ${ }^{18}$. Ha sido ampliamente validado y se encuentra traducido a varios idiomas. Incluye 25 ítems que representan problemas emocionales, vitalidad, dormir, comer / beber, y el funcionamiento físico / social ${ }^{19}$. A cada ítem se le asigna un puntaje entre 1 y 7 . Cuenta con validación de constructo (estudiada con constructos similares [SF-36 y GSRS]) y consistencia interna $\geq 0,89$. Respecto de dispepsia y trastornos gastrointestinales funcionales, la escala se comporta de forma similar, con excepción de pacientes con endoscopia normal, en los que la escala tiene un desempeño peor.

\section{REFLUX trial group}

Creado por investigadores del Centre for Health Economics, de la Universidad de York y publicado en $2007^{20}$. En su génesis, se realizaron grupos focales con pacientes con ERGE en tratamiento médico o quirúrgico. Está compuesto por 31 ítems distribuidos en 7 dominios (pirosis, reflujo ácido, "vientos"; alimentación y deglución; movilidad intestinal, sueño, trabajo; y actividades sociales y físicas). Cuenta con estudios de consistencia interna, validez de criterio (respecto del SF-36) y sensibilidad al cambio en relación a la administración de medicación con seguimiento de tres meses.

\section{Visick grading system}

Publicada en 1948, fue creada con el objetivo de medir resultados postoperatorios de la gastrectomía radical por ulcus péptico ${ }^{17}$. Posteriormente, su aplicación se amplió para valorar CV en pacientes sometidos a cirugía por úlcera péptica en general ${ }^{21-23}$; y luego para evaluación de resultados de diversos tipos de cirugías gástricas ${ }^{24,25}$. Desde hace un tiempo se ha utilizado para evaluar la apreciación de los pacientes respecto a la cirugía antirreflujo, tanto por vía abierta ${ }^{26}$ como laparoscópica ${ }^{27}$. Se describe que aunque puede ser aplicada para monitorizar efectos subjetivos de la cirugía antirreflujo dada la buena correlación entre síntomas cardinales de ERGE, es difícil categorizar adecuadamente los resultados en cuanto a síntomas post cirugía.

\section{Relacionados con la obesidad y cirugía bariátrica}

\section{BAROS (Bariatric Analysis and Reporting Outcome System)}

Generado por académicos del Spring Branch Medical Center, en 1998 con el objetivo de valorar resultados en cirugía bariátrica. Consta de una tabla que incluye tres columnas con los principales dominios de análisis: pérdida de peso, mejora de condiciones médicas y $\mathrm{CV}^{28,29}$. El dominio mejora de condiciones médicas, evalúa las comorbilidades que presenta cada paciente y compara la gravedad de estas pre y post cirugía; para lo cual, se definieron por consenso 7 patologías como comorbilidades mayores (hipertensión arterial, enfermedad cardiovascular, dislipidemia, diabetes mellitus tipo II, síndrome de apnea e hipopnea obstructiva del sueño, osteoartritis e infertilidad); cuyas categorías de respuesta son mejoría (se puede controlar con una disminución de dosis) o resolución (no se requiere medicación para su control). El dominio 
$\mathrm{CV}$, corresponde al cuestionario Moorehead-Ardelt que evalúa autoestima, actividad física, vida social, condiciones de trabajo y actividad sexual ${ }^{30}$; usando dibujos simples para ofrecer opciones de respuesta en cada uno de los 5 ítems; de forma tal que en este dominio, se considera prioritario el análisis de la autoestima, razón por la que a diferencia de los otros 4 aspectos evaluados, autoestima tiene un máximo de 1 punto, en cambio las otras sólo 0,5 . En cada dominio, se otorga un puntaje de -1 a 3 puntos, siendo un puntaje negativo reflejo de fracaso. Posteriormente, se suma el número de puntos de cada columna. La presencia de complicaciones mayores o menores o la necesidad de re-intervención son consideradas, debiéndose restar al puntaje total el número de puntos según sea el caso. El puntaje final permite incluir al paciente dentro de 1 de los 5 grupos que definen el concepto de éxito o fracaso. En la eventualidad de realizar evaluación a pacientes sin comorbilidad previa a la cirugía, el sistema debe ser adaptado antes de calcular los resultados. La utilización de este cuestionario en población Europea demostró ser válido y confiable.

\section{BQLI (Bariatric Quality of Life Index)}

Diseñado por académicos del Obesity Academy Frankfurt en 2005, con el objetivo de valorar resultados en cirugía bariátrica. Fue desarrollado a partir de sugerencias y comentarios realizados por un grupo de pacientes a los que se aplicó los cuestionarios SF-36 y BAROS. Se elaboró un instrumento preliminar, que fue posteriormente aplicado a 110 pacientes, y adaptado antes de su elaboración final. Está compuesto por 19 ítems que evalúan bienestar psicológico, funcionalidad social y física, problemas y síntomas relacionados con la cirugía y comorbilidades. Cada ítem tiene un puntaje asignado. El puntaje final es de 0 a 78 puntos, reflejando a menor puntaje, peor resultado. Se encuentra actualmente validado en su versión original en alemán ${ }^{31,32}$.

\section{IWQoL (Impact of Weight on Quality of Life)}

Creado por académicos del Departmento de Medicina Familiar y Comunitaria de la Universidad de Duke y publicado en su primera versión en $1995^{33}$. Es el primer instrumento desarrollado para evaluar $\mathrm{CV}$ en obesos (impacto del peso en la CV). Sus objetivos son medir la forma en que el peso afecta la $\mathrm{CV}$, determinar los aspectos de CV que son más afectados por el peso; y medir los avances en CV asociados a la pérdida de peso. Compuesto por 74 ítems que evalúan 8 dominios: salud (14), social/ interpersonal (11), trabajo (7), movilidad (10), autoestima (10), vida sexual (6), actividades de la vida diaria (7) y satisfacción con la comida (9). Su proceso de validación se publicó en 1997, y cuenta con estudios de validez de constructo, consistencia interna y confiabilidad test re-test ${ }^{34}$. El inconveniente es la extensión del instrumento, la que pudiese influir en la actitud y respuestas de los sujetos.

\section{IWQoL-Lite (Impact of Weight on Quality of Life-Lite)}

Generado por académicos de la Obesity and Quality of Life Consulting de Durham, y publicada en 2002. Fue construido a partir del IWQoL con el objetivo de generar un instrumento abreviado del original de 74 ítems. Está validado y es psicométricamente más sólido y clínicamente más sensible que el original. Está compuesto por 31 ítems de auto-reporte, cada una de las cuales se califica entre 1 ("nunca cierto") y 5 ("siempre cierto"). Se obtienen puntajes específicos en cinco dominios: estado físico (11 ítems), autoestima ( 7 ítems), vida sexual (4 ítems), ansiedad en público (5 ítems) y trabajo (4 ítems). Los puntajes más altos se asocian con menor compromiso de la CVRS (mejor CV). En su versión original (inglés), mostro tener buena consistencia interna $(\geq 0,90)$, confiabilidad test-retest $(\geq 0,83)$ y sensibilidad al cambio. En un estudio colombiano se reportó una consistencia interna con alfa de Cronbach $\geq 0,89$; y una confiabilidad test-retest con coeficiente de correlación intraclase $\geq 0,85^{35,36}$.

\section{Relacionados con cánceres del tubo digestivo alto}

\section{EORTC QLQ-30}

Elaborado por la organización europea para la investigación y tratamiento del cáncer (EORTC) en 1993. Se encuentra validado y traducido a 43 idiomas. Tiene una alta correlación con la clínica; sin embargo, necesita de la aplicación de módulos complementarios. Está compuesto por 30 ítems que se agrupan en 5 dominios (funcionamiento físico, social, emocional, cognitivo y rol); tres escalas de síntomas (fatiga, dolor, náuseas y vómitos); una escala global de salud/CV y un número de ítems individuales relacionados con los síntomas de la enfermedad y su tratamiento, así como un ítem de impacto económico. Las respuestas a los ítems de las escalas se refieren a "la semana pasada," excepto la escala de funcionamiento físico cuyo marco temporal es el presente. Dichas respuestas obedecen a un formato tipo Likert que oscila entre 1 ("en absoluto") y 4 ("mucho"), excepto en la escala global que tiene siete opciones de respuesta. Todas las escalas e ítems individuales se convierten en una puntuación de 0 a 100 . Ha sido complementado con ítems específicos para cada tipo de cáncer, lo que permite añadir una sensibilidad necesaria para detectar variaciones en la CVRS de los pacientes ${ }^{37,38}$. 


\section{EORTC OES-18}

Generado por académicos del Departamento de Cirugía de la Universidad de Bristol Royal Infirmary en 1996. Es un módulo que complementa EORTC QLQ-C30, específico para neoplasias esofágicas, que se compone por 18 ítems, relacionadas con la capacidad de comer y beber, sabor de boca, hablar, toser, dolor y síntomas de reflujo; que tienen 4 opciones de respuesta tipo escala Likert ${ }^{39}$. La adición del módulo específico para cáncer de esófago al cuerpo del cuestionario mejora la sensibilidad y especificidad del instrumento para la detección incluso de pequeños beneficios relacionados con nuevas modalidades de tratamiento. Cuenta con una adaptación al español realizada en Colombia ${ }^{40}$.

\section{EORTC QLQ-30 STO22}

Creado por la EORTC como suplemento al QLQ-C30 en 2001 para mejorar la sensibilidad y especificidad para detectar variaciones en la CVRS de pacientes con cáncer gástrico ${ }^{41}$. Está compuesto por 22 ítems agrupados en 5 dominios (disfagia, dolor, dieta, síntomas y problemas emocionales) y 4 ítems específicos (boca seca, sabor, imagen corporal y caída del cabello), relacionados con los síntomas de la enfermedad, efectos colaterales del tratamiento y temas emocionales específicos; que tienen 4 opciones de respuesta tipo escala Likert. Cuenta con estudios de validez psicométrica y clínica lo que apoya su uso en pacientes con cáncer gástrico sometidos a cirugía, cirugía y quimioterapia, quimioterapia paliativa, cirugía paliativa o cuidados paliativos ${ }^{42}$.

\section{EORTC QLQ-30 OG25}

Diseñado por la EORTC como suplemento al QLQ-C30 para evaluar CVRS en pacientes con cáncer del esófago, unión gastroesofágica y estómago. Se generó a partir de los cuestionarios de EORTC QLQ-STO22 y QLQ-OES18 y se publicó en 2007. Se compone de 25 ítems agrupados en 6 dominios: disfagia, restricciones para alimentarse, reflujo, odinofagia, dolor y ansiedad; que tienen 4 opciones de respuesta tipo escala Likert. Cuenta con estudios de validez y confiabilidad. Se ha verificado que permite distinguir localizaciones tumorales y etapas de enfermedad ${ }^{43,44}$.

\section{FACT-G (Functional Assessment of Cancer Therapy-General Questionnaire)}

Creado por investigadores del Rush-PresbyterianSt Luke's Medical Center, para evaluar CV del paciente oncológico, fue publicado en 1993. Comprende 27 ítems que evalúan 4 dominios sobre $\mathrm{CV}$ : física, social /familiar, emocional y bienestar funcional. Cada dimensión se evalúa con puntaje de 0 (en absoluto) a 4 (mucho). El puntaje total es la suma de los puntajes de los cuatro dominios mencionados y va desde 0 a $180^{45}$. Datos previos sobre estudios con FACT-G (versión española 2) aplicadas en pacientes uruguayos con cáncer sugirieron que su confiabilidad era aceptable en todos los ítems excepto en el dominio emocional por lo que surgió la interrogante sobre si estos dominios tenían suficiente consistencia interna para justificar su uso independiente de las culturas y si había equivalencia del dominio emocional versión española e inglesa ${ }^{46}$. Desde entonces, se ha estado revisando el cuestionario (versión en español 4), al que se adicionó el ítem "me preocupa que mi enfermedad empeore" en la evaluación del área emocional; se eliminaron 2 ítems del dominio "relación con el médico" y se redactaron nuevamente los 12 ítems en español para mejorar su legibili$\mathrm{dad}^{47}$. Este se decidió incorporar por estar asociado a la medición de CVRS en pacientes con cánceres digestivos. Se encuentra ampliamente validado, tiene alta correlación con la clínica, pero necesita de módulos complementarios.

\section{FACT-E}

Generado en la División de Cirugía Torácica de la Universidad de Toronto, y publicado en 2006. Es un módulo específico para neoplasias esofágicas que se complementa con FACT- $\mathrm{G}^{48}$. Consta de estudios de consistencia interna (alfa de Cronbach > 0,70); validez convergente (comparado con EORTC QLQ30 y OES 24), respuesta al cambio (en especial en sujetos que reciben radioquimioterapia preoperatoria). Compuesto por 17 ítems, agrupados en 5 dominios (físico, funcional, social, emocional; y de síntomas relacionados con cáncer de esófago); todos con 5 opciones de respuesta, tipo Likert.

\section{FACT-Ga}

Creado por académicos del Departamento de Psicología de la Universidad de Calgary, y publicado en 2011. Es un módulo que complementa FACT-G específico para neoplasias gástricas que se complementa con FACT- $\mathrm{G}^{49}$. Consta de estudios de consistencia interna (alfa de Cronbach $>0,70$ ); validez convergente (comparado con SF-36), validez de criterio, sensibilidad al cambio. Compuesto por 47 ítems, agrupados en 5 dominios (físico, social, emocional, funcional; y de síntomas relacionados con cáncer gástrico).

\section{KPS (Karnofsky perfomance status)}

Diseñado por Karnofsky y Burchenal en 1949 para evaluar la capacidad de un paciente oncológico para sobrevivir a una quimioterapia. Ha sido latamente utilizado, pero su validación data sólo de $1984^{50}$, existiendo una fuerte correlación con una serie de variables relacionadas con las funciones 
físicas del paciente. La evaluación se hace en rangos de $100 \%$ a $0 \%$ (correspondiendo el $100 \%$ a normal, asintomático; y el $0 \%$ a muerte); lo que se dispone en tres posibles subgrupos: "capaz de realizar una actividad y trabajo normal; sin necesidad de cuidados especiales" (100\% a $80 \%)$; "incapaz de trabajar, pero capaz de vivir en el hogar y atender la mayoría de las necesidades personal" (70\% a 50\%); "imposibilidad de cuidarse a sí mismo, requiere de atención institucional $u$ hospitalaria equivalente, la enfermedad puede progresar rápidamente" $(40 \%$ a $0 \%)$. Se ha visto que puede predecir correctamente la muerte precoz; sin embargo, altos puntajes iniciales no han predicho una larga supervivencia. El deterioro del paciente con la subsecuente muerte en pocos meses puede ser predicho por una rápida caída del puntaje de Karnofsky. Debe considerarse que debido a que este índice pone énfasis en el rendimiento físico y dependencia del paciente, puede develar una visión errónea respecto de la esfera psicológica y social del paciente, conduciendo a error en la medición de $\mathrm{CV}$ del paciente.

\section{RSCL (Rotterdam Symptom Checklist)}

Generado por investigadores del Departamento de Oncología Clínica de la Universidad de Leiden y publicado en $1990^{51}$. Su objetivo es medir el malestar físico y psicológico de pacientes con cáncer en general. Compuesta por 27 ítems que se distribuyen en dos dominios. En su diseño se aplicó un análisis de componentes principales en tres estudios (quimioterapia y seguimiento; quimioterapia por cáncer avanzado de ovario; y pacientes oncológicos sin tratamiento, en etapa libre de enfermedad y "controles sanos"); lo que permitió definir la estabilidad del instrumento en diferentes poblaciones. Cuenta además con estudio de consistencia interna (alfa de Cronbach $\geq 0,88)$ y confiabilidad $(\geq 0,71)$. Consta de 39 ítems, agrupados en 4 dominios (síntomas de angustia física, de angustia sicológica, nivel de actividad, evaluación global de vida).

\section{Discusión}

Si bien es cierto que en este manuscrito se consideraron algunos instrumentos no directamente concernientes con cirugía digestiva alta; como IWQoL, IWQoL-Lite, KPS, RSCL; decidimos incluirlos porque todos ellos son instrumentos específicos (no genéricos para medición de $\mathrm{CV}$ ); y porque además, han sido utilizados con frecuencia en estudios relacionados. Los dos primeros en estudios que reportan resultados en cirugía bariátrica; y los segundos en estudios de cáncer esofágico, esófago-gástrico y gástrico.
Resulta interesante conocer estas herramientas, casi todas validadas de forma adecuada; y que, si bien es cierto se utilizan de forma habitual en investigación clínica, no son demasiado empleados con estos propósitos en nuestro medio aún.

Por otro lado, es interesante saber que se dispone de más de uno para medir hechos similares, lo que permite al investigador la opción de escoger el instrumento que más se ajusta a su realidad clínica, así como al propósito de su investigación. Por lo demás, no todos se encuentran traducidos al español, hecho fundamental a la hora de decidir cuál utilizar.

Otro hecho interesante, es conocer aquellos no traducidos al español, pues es una idea de investigación a considerar, pues el proceso de traducción lingüística de un instrumento es una línea de investigación atractiva de considerar por los distintos grupos de trabajo.

A modo de conclusión, podemos señalar que existe una variedad interesante de instrumentos para medir CVRS en relación con patología digestiva alta, tanto de tratamiento médico como quirúrgico; y que el conocerlos constituye una ayuda al clínico (en este caso para el cirujano) en el desarrollo de su práctica quirúrgica cotidiana; la toma de decisiones en salud; y la conducción de procesos de investigación clínica, en los cuales pueden ser usados como instrumentos de medición.

\section{Referencias}

1. Yates JW, Chalmer B, McKeqner FP. Evaluation of patients with advanced cancer using the Karnofsky performance status. Cancer 1980;45:2220-4.

2. Joan Monés. “¿Se puede medir la calidad de vida? ¿Cuál es su importancia?”. Cir Esp. 2004;76:71-7.

3. Guyatt G, Feeny DH, Patrick DL. Measuring HealthRelated Quality of Life. Ann Intern Med. 1993;118:6229.

4. Brundage M, Blazeby J, Revicki D, Bass B, de Vet H, Duffy $\mathrm{H}$, et al. Patient-reported outcomes in randomized clinical trials: development of ISOQOL reporting standards. Qual Life Res. 2013;22:1161-75.

5. Manterola C, Urrutia S, Otzen T. Calidad de vida relacionada con salud. Una variable resultado a considerar en investigación clínica. Int J Morphol. 2013;31:151723.

6. ScienceCentral.com. Documents in Information Science" (DoIS). http://www.sciencecentral.com/site/518669. Consultado el 30 de abril de 2013.

7. Velanovich V, Karmy-Jones R. Measuring gastroesophageal reflux disease: relationship between the Health-Related Quality of Life score and physiologic parameters. Am Surg. 1998;64:649-53.

8. Chan Y, Ching JY, Cheung CM, Tsoi KK, Polder-Verkiel 
S, Pang SH, et al. Development and validation of a disease-specific quality of life questionnaire for gastrooesophageal reflux disease: the GERD-QOL questionnaire. Aliment Pharmacol Ther. 2010;31:452-60.

9. Holtmann G1, Chassany O, Devault KR, Schmitt H, Gebauer U, Doerfler H, et al. International validation of a health-related quality of life questionnaire in patients with erosive gastro-oesophageal reflux disease. Aliment Pharmacol Ther. 2009;29:615-25.

10. Eypasch E, Williams JI, Wood-Dauphinee S, Ure BM, Schmulling C, Neugebauer E, et al. Gastrointestinal Quality of Life Index: development, validation and application of a new instrument. Br J Surg. 1995;82:216-22.

11. Quintana JM, Cabriada J, López de Tejada I, Varona M, Oribe V, Barrios B, y cols. Traducción y validación del Índice de Calidad de Vida Gastrointestinal (GIQLI). Rev Esp Enferm Dig. 2001;93:693-9.

12. Svedlund J, Sjödin I, Dotevall G. GSRS-A Clinical Rating Scale for Gastrointestinal Symptoms in Patients with Irritable Bowel Syndrome and Peptic Ulcer Disease. Dig Dis Sci. 1988;33:129-34.

13. Kulich KR, Malfertheiner P, Madisch A, Labenz J, Bayerdörffer E, Miehlke S, et al. Psychometric validation of the German translation of the Gastrointestinal Symptom Rating Scale (GSRS) and Quality of Life in Reflux and Dyspepsia (QOLRAD) questionnaire in patients with reflux disease. Health Qual Life Outcomes 2003;1:62.

14. Asberg M, Montgomery SA, Perris C, Schalling D, Sedvall G. A comprehensive psychopathological rating scale. Acta Psychiatr Scand Suppl. 1978;271:5-27.

15. Chassany O1, Marquis P, Scherrer B, Read NW, Finger T, Bergmann JF, et al. Validation of a specific quality of life questionnaire for functional digestive disorders. Gut 1999;44:527-33.

16. Zéman Zs, Rózsa S, Tarkó E, Tihanyi T. Psychometric documentation of a quality-of-life questionnaire for patients undergoing antireflux surgery (QOLARS). Surg Endosc. 2005;19:257-61.

17. Visick AH. Measured radical gastrectomy; review of 505 operations for peptic ulcer. Lancet 1948;1 (6501):551-5

18. Wiklund IK, Junghard O, Grace E, Talley NJ, Kamm M, Veldhuyzen van Zanten S, et al. Quality of Life in Reflux and Dyspepsia patients. Psychometric documentation of a new disease-specific questionnaire (QOLRAD). Eur J Surg Suppl. 1998;583:41-9.

19. Kulich KR, Madisch A, Pacini F, Piqué JM, Regula J, Van Rensburg CJ, et al. Reliability and validity of the Gastrointestinal Symptom Rating Scale (GSRS) and Quality of Life in Reflux and Dyspepsia (QOLRAD) questionnaire in dyspepsia: A six-country study. Health Qual Life Outcomes 2008;6:12.

20. Macran S, Wileman S, Barton G, Russell I. The development of a new measure of quality of life in the management of gastro-oesophageal reflux disease: the Reflux questionnaire. Qual Life Res. 2007;16:331-43.

21. Selking O, Krause U, Nilsson F, Thorén L. Parietal cell vagotomy and truncal vagotomy as treatment of duodenal ulcer. A prospective randomized trial. Acta Chir Scand. 1981;147:561-7.

22. Byrne DJ, Brock BM, Morgan AG, McAdam WA. Highly selective vagotomy: a 14-year experience. $\mathrm{Br} \mathrm{J}$ Surg. 1988;75:869-72.

23. Stanton PD, Anderson JR. Results of surgery for duodenal ulcer: assessment by patients. $\mathrm{Br} \mathrm{J}$ Surg. 1991;78:815-7.

24. Speranza V, Basso N, Lezoche E, Materia A, Bagarani M, Paduos A. Management and long-term results in patients with two-thirds gastrectomy and stomal ulcer. Am J Surg. 1981;141:105-10.

25. Siedek M, Lindecken KD, Birtel FJ, Rubinger M, Ebener J. B II-Y-Roux anastomosis-resection procedure of choice in complicated ulcer? Langenbecks Arch Chir. 1984;362:61-8

26. Bell RC, Hanna P, Powers B, Sabel J, Hruza D. Clinical and manometric results of laparoscopic partial (Toupet) and complete (Rosetti-Nissen) fundoplication. Surg Endosc. 1996;10:724-8.

27. Dallemagne B, Weerts JM, Jeahes C, Markiewicz S. Results of laparoscopic Nissen fundoplication. Hepatogastroenterology 1998;45:1338-43.

28. Oria HE, Moorehead MK. Bariatric Analysis and Reporting Outcome System (BAROS). Obes Surg. 1998;8:487-99.

29. Wolf AM, Falcone AR, Kortner B, Kuhlmann HW. "BAROS: An Effective System to Evaluate the Results of Patients after Bariatric Surgery". Obes Surg. 2010;10:445-50.

30. Moorehead MK, Ardelt-Gattinger E, Lechner H, Oria HE. The Validation of the Moorehead-Ardelt Quality of Life Questionnaire II. Obes Surg. 2013;13:684-92.

31. Weiner S, Sauerland S, Fein M, Blanco R, Pomhoff I, Weiner RA. The Bariatric Quality of Life (BQL) Index: A Measure of Well-being in Obesity Surgery Patients. Obes Surg. 2005;15:538-45.

32. Weiner S, Sauerland S, Weiner R, Cyzewski M, Brandt J, Neugebauer E. Validation of the Adapted Bariatric Quality of Life Index (BQL) in a Prospective Study in 446 Bariatric Patients as One-Factor Model. Obes Facts 2009;2 Suppl 1:63-6.

33. Kolotkin RL, Head S, Hamilton M, Tse CKJ. Assessing impact of weight on quality of life. Obesity Res. 1995;3:49-56.

34. Kolotkin RL, Head S, Brookhart A. Construct Validity of the Impact of Weight on Quality of Life Questionnaire. Obes Res. 1997;5:434-41.

35. Kolotkin RL, Crosby RD. Psychometric evaluation of the impact of weight on quality of life-lite questionnaire (IWQOL-Lite) in a community simple. Qual Life Res. 2002;11:157-71. 
36. Brazier JE, Kolotkin RL, Crosby RD, Williams R. Estimating a Preference-Based Single Index for the Impact of Weight on Quality of Life-Lite (IWQOL-Lite) Instrument from the SF-6D. Value Health 2004;7:490-8.

37. Aaronson NK, Ahmedzai S, Bergman B, Bullinger M, Cull A, Duez NJ, et al. The European Organization for Research and Treatment of Cancer QLQ-C30: a qualityof-life instrument for use in international clinical trials in oncology. J Natl Cancer Inst. 1993;85:365-76.

38. Vickery CW, Blazeby JM, Conroy T, Arraras J, Sezer O, Koller M, et al; EORTC Quality of Life Group. Developmen of an EORTC disease-specific quality of life module for use in patients with gastric cancer. Eur $\mathrm{J}$ Cancer 2001;37:966-71.

39. Blazeby JM, Alderson D, Winstone K, Steyn R, Hammerlid E, Arraras J, et al. Development of an EORTC questionnaire module to be used in quality of life assessment for patients with oesophageal cancer. The EORTC Quality of Life Study Group. Eur J Cancer 1996;32A:1912-7.

40. Ibáñez C, Sierra F, Sánchez R. Adaptación transcultural del cuestionario EORTC QLQ-OES18 para medir calidad de vida en pacientes con cáncer de esófago en Colombia. Rev Colomb Cancerol. 2013;17:69-76.

41. Vickery CW, Blazeby JM, Conroy T, Arraras J, Sezer O, Koller M, et al. Development of an EORTC diseasespecific quality of life module for use in patients with gastric cancer. Eur J Cancer. 2001;37:966-71.

42. Blazeby JM, Conroy T, Bottomley A, Vickery C, Arraras J, Sezer O, et al; European Organization for Research and Treatment of Cancer Gastrointestinal and Quality of Life Groups. Clinical and psychometric validation of a questionnaire module, the EORTC QLQ-STO 22, to assess quality of life in patients with gastric cancer. Eur J Cancer 2004;15:2260-8.

43. Lagergren P, Fayers P, Conroy T, Stein HJ, Sezer O, Hardwick R, et al; European Organization for Research Treatment of Cancer Gastrointestinal and Quality of Life Groups. Clinical and psychometric validation of a questionnaire module, the EORTC QLQ-OG25, to as- sess health-related quality of life in patients with cancer of the oesophagus, the oesophago-gastric junction and the stomach. Eur J Cancer. 2007;43:2066-73.

44. Oñate-Ocaña LF, Velázquez-Monroy $\mathrm{N}$, Vázquez L, Espinosa-Mireles-de-Villafranca P, Núñez-Rosas E, et al. Clinical validation of the EORTC QLQ-OG25 questionnaire for the evaluation of health-related quality of life in Mexican patients with esophagogastric cancers. Psychooncology 2012;21:745-53.

45. Cella DF, Tulsky DS, Gray G, Sarafian B, Linn E, Bonomi A, et al. The Functional Assessment of Cancer Therapy scale: development and validation of the general measure. J Clin Oncol. 1993;11:570-9.

46. Dapueto JJ, Francolino C, Servente L, Chang C, Gotta I, Levin R, et al. Evaluation of the Functional Assessment of Cancer Therapy-General (FACT-G) Spanish Version 4 in South America: Classic Psychometric and Item Response Theory Analyses. Health Qual Life Outcomes 2003;1:32.

47. Dapueto JJ, Francolino C, Gottab I, Levin R, Alonso I, Barrios E, et al. Evaluation of the functional assesment of cancer therapy-general questionnaire (FACT-G) in South American Spanish speaking population. PsychoOncology 2010;10:88-92.

48. Darling G, Eton DT, Sulman J, Casson AG, Celia D. Validation of the functional assessment of cancer therapy esophageal cancer subscale. Cancer 2006;107:854-63.

49. Garland SN, Pelletier G, Lawe A, Biagioni BJ, Easaw J, Eliasziw M, et al. Prospective evaluation of the reliability, validity, and minimally important difference of the functional assessment of cancer therapy-gastric (FACTGa) quality-of-life instrument. Cancer 2011;117:130212.

50. Schaq CC, Heinrich RL, Ganz PA. Karnofski perfomance status revisited: reliability, validity, and guidelines. J Clin Oncol 1984;2:187-93.

51. de Haes JC, van Knippenberg FC, Neijt JP. Measuring psychological and physical distress in cancer patients: structure and application of the Rotterdam Symptom Checklist. Br J Cancer 1990;62:1034-8. 